\title{
Carcinomatous Myelitis and Meningitis after a Squamous Cell Carcinoma of the Lip
}

\author{
Isabelle Pougnet $^{a} \quad$ Anne Murati ${ }^{b-d, f}$ Anthony Sarran ${ }^{c}$ \\ Patrice Viens ${ }^{a, d, e, f} \quad$ Renaud Sabatier ${ }^{a, d, e, f}$ \\ Departments of ${ }^{a}$ Medical Oncology, ${ }^{b}$ Pathology, and ${ }^{c}$ Radiology, Institut Paoli-Calmettes, \\ ${ }^{\mathrm{d}}$ Department of Molecular Oncology, INSERM U1068, Centre de Recherche en \\ Cancérologie de Marseille, ${ }^{e}$ CNRS UMR7258, CRCM, and ${ }^{f}$ University of Aix-Marseille, \\ Marseille, France
}

\section{Key Words}

Head and neck carcinoma $\cdot$ Squamous cell $\cdot$ Meningitis · Spinal cord metastasis

\begin{abstract}
Background: Nervous central system metastases from head and neck squamous cell carcinoma (SCC) are rare. We report an exceptional case of isolated leptomeningeal and spinal cord involvement few years after the diagnosis of invasive SCC of the lip. Case Report: A 33-year-old man with a history of infracentimetric carcinoma of the lip developed back pain associated with progressive neurological disorders leading to paraplegia. This atypical presentation led to initial misdiagnosis, but radiological and cytological explorations finally confirmed the diagnosis of leptomeningeal and intramedullar secondary spinal cord lesions from his previously treated head and neck SCC. Systemic targeted therapy with epidermal growth factor receptor inhibitor and intrathecal chemotherapy led to prolonged disease stabilization. Conclusion: To our knowledge, this is the first case of isolated neurological metastases from a head and neck SCC. Combination of systemic targeted therapy and intrathecal chemotherapy may be effective in such cases.

(c) 2014 S. Karger AG, Basel
\end{abstract}

\section{Introduction}

Head and neck squamous cell carcinoma (SCC) is the sixth cause of cancer worldwide in males and the fifth in France [1]. Most of the patients can be cured with combinations of surgery, radiation therapy, and /or chemotherapy. Distant metastases from head and neck 
Pougnet et al.: Carcinomatous Myelitis and Meningitis after a Squamous Cell Carcinoma of the Lip

SCC often involve lungs, bones or liver. Central nervous system lesions have been scarcely described. Among these lesions, carcinomatous meningitis (CM) is a rare phenomenon and very few cases have been reported [2]. Secondary intramedullary spinal cord lesions are rare, with only one case described in the literature [3]. Here, we report the case of a 33-yearold man with intramedullary spinal cord and leptomeningeal involvement secondary to a SCC of the lip.

\section{Case Report}

The patient was a 33-year-old Caucasian man without any specific personal or familial history. He was an active smoker without alcoholic intoxication. In 2009, he presented a lesion of the lower lip. He underwent resection of this lesion, and the first pathological analysis revealed a salivary ductal ecstasy.

One year later, he developed a peripheral facial paralysis associated with left trigeminal neuralgia. MRI showed perineural invasion of the trigeminal nerve within the temporal fossa (fig. 1). Surgical biopsies performed in July 2010 identified secondary lesions from a welldifferentiated SCC localized around the V and VII nerve sheaths. According to this diagnosis, a new pathological analysis of the previous resection of the lip was performed in a cancer reference center and diagnosis of SCC of the lip was made. The patient started radiochemotherapy in September 2010. He received 70 Gy in 35 fractions, and five courses of an association of 5-fluorouracil and cisplatinum. First radiological assessments, 4 months after the end of radio-chemotherapy showed no residual disease.

However, in November 2011, the patient presented with new neurological symptoms: paraparesis and back pain. MRI showed medullary myelitis without meningeal enhancement. Lumbar puncture revealed inflammatory cells without atypical cells within the cerebrospinal fluid (CSF). Cerebral MRI and positron emission tomography scan were normal with no evidence of visceral metastasis. Multiple sclerosis was suspected and the patient received several corticosteroid flashes who led to partial improvement of symptoms. Nevertheless, other explorations were negative, and it was not possible to certify the diagnosis of multiple sclerosis. In early 2012, the patient relapsed and developed paraparesis once again. Sequential lumbar punctures found no abnormal cells. He was given a symptomatic treatment combining corticosteroid and plasma depletion. In March 2012, CSF analysis showed intrathecal immunoglobulin syntheses with oligoclonal immunoglobulin bands. Medullary MRI revealed intramedullary hyperintensity without enhancement after gadolinium injection. The diagnosis of dysimmune myelitis was made and the patient received an immunosuppressive drug (mycophenolate mofetil). It is of note that his neurological symptoms partially improved under this treatment.

In August 2012, he subsequently developed progressive back pain, sphincter disorders, and lower limb weakness. A new MRI performed in November showed enhancement of the meningeal nodules and abnormal leptomeningeal contrast enhancement (fig. 2). There was no evidence of brain lesions. New lumbar punctures revealed several atypical cells consistent with metastatic squamous cells (fig. 3) associated with low glycorrhachia and high proteinorrhachia levels $(1.25 \mathrm{~g} / \mathrm{l})$. These cells were well-differentiated with nuclear abnormalities consistent with meningeal infiltration by a well-differentiated SCC. Intrathecal immunoglobulin synthesis was no longer seen. Moreover, chest and abdominal CT-scans did not identify other secondary lesions, especially bone metastasis. Nevertheless, a PET scan showed an intramedullary spinal cord metabolism enhancement. All these data were consistent with the diagnosis of CM associated with a carcinomatous myelitis. 
Pougnet et al.: Carcinomatous Myelitis and Meningitis after a Squamous Cell

The patient underwent intrathecal weekly infusions of methotrexate $(15 \mathrm{mg}$ ) and methylprednisolone combined with systemic treatment based on carboplatin every 3 weeks and weekly cetuximab $\left(250 \mathrm{mg} / \mathrm{m}^{2}\right)$. After two cycles, the disease was clinically stable with no extension of symptoms, the proteinorrhachia level was still high $(1.5 \mathrm{~g} / \mathrm{l})$, and metastatic cells were still identified in the CSF. However, MRI showed a slight amelioration and cytological analysis revealed only isolated metastatic cells. A new chest and abdominal CTscan showed no other secondary lesions. As the patient developed a febrile neutropenia, we decided to stop systemic chemotherapy. He received cetuximab $500 \mathrm{mg} / \mathrm{m}^{2}$ and intrathecal methotrexate $15 \mathrm{mg}$ every 2 weeks. After three courses of treatment, clinical and MRI evaluation showed no neurological worsening and a stabilization of the medullar lesions. These observations were confirmed 6 months after treatment start.

\section{Discussion}

To our knowledge, we have described the first case of well-differentiated SCC with an early and isolated intramedullary and meningeal relapse in a young Caucasian patient.

Head and neck SCC are frequent but curable diseases. Distant metastases are rare and cannot be cured. CM, or leptomeningeal infiltrate by neoplastic cells, is a rare but lethal localization of this kind of tumor. The prognosis is very poor because this complication remains incurable. It occurs in 5-10\% of solid tumors [4], and it is more frequent in small cell lung cancer, breast cancer or melanoma, and the most common symptoms are headaches, nausea, vomiting and cognitive disorder. In head and neck cancer, it frequently involves the cranial nerves, and mainly the optic nerve [5, 6]. Neurological symptoms and malignant cells in CSF are two essential elements for diagnosis. However, the first CSF examination can be negative in 50\% of cases [5]. In those cases, other abnormal features such as increase of protein levels and low glycorrhachia can help physicians to make this diagnosis [2]. The most sensitive imagery is currently MRI that can show nodules or meningeal contrast enhancement [7]. The more common treatments of CM are currently iterative intrathecal chemotherapy like methotrexate and Ara-C or intraventricular injection through Ommaya reservoir $[2,8]$. However, there is no uniform approach in meningitis treatment because of limited recruitment of these patients in clinical trials. Thus, no straight forward guidelines exist in the literature.

Leptomeningeal metastases from head and neck SCC have been scarcely described in the literature. One case was reported in 1984 [6]: a 70-year-old man who developed a meningeal spread of a SCC of the lower lip associated to an ipsilateral mandible metastasis. The treatment consisted of intermediate doses of intravenous methotrexate with citrovorum and weekly administrations of intravenous methotrexate and bleomycin, showing no effect on his clinical course. He died 3 years after the first diagnosis of SCC and 1 year after meningitis discovery. A review of literature made in 1986 found only two other case reports [2]. Three other cases had been reported since this date. The first one was described in 2003 and concerned a 51-year-old man who developed a left supraglottic SCC [9]. He subsequently presented lower back pain, and the diagnosis of leptomeningeal metastasis was made on a lumbar puncture. He underwent radiotherapy and intrathecal chemotherapy. He finally died 3 weeks after completing radiotherapy. Another case, published in 2005, was about a 43year-old woman who presented a nasopharyngeal SCC in 1985 [10]. Eight years later, she developed progressing neural disorders leading to the diagnosis of CM. She was treated by systemic chemotherapy including methotrexate, 5-fluorouracil and leucovorin. She died 3 years after the diagnosis of meningitis. The most recent case was published in 2006, and 
Pougnet et al.: Carcinomatous Myelitis and Meningitis after a Squamous Cell Carcinoma of the Lip

described a CM of the lips due to SCC in a 51-year-old man. He received whole spine radiation therapy and dexamethasone but died 3 months after completing radiotherapy [5].

Moreover, it is of note that only one case of intramedullary spinal cord metastasis has been published [3]. A 36-year-old woman with tongue SCC developed multiple intramedullary spinal cord metastases few months after a skull base relapse. She was treated by radiation therapy without any clinical improvement. No meningeal invasion was noted in this report.

Cetuximab is an antibody targeting the epidermal growth factor receptor. It is a highweight molecule usually described to not cross the blood brain barrier [11]. However, data showing that it could have a clinical activity in case of central nervous system involvement by neoplastic diseases exist. Cetuximab has been described to enhance survival when administered in monotherapy and to be synergic with radiation therapy in mice presenting glioblastoma multiforme [12]. Moreover, the use of radiolabeled cetuximab in a patient with lung SCC showed an accumulation of the molecule in brain lesions, suggesting that it can cross the blood brain barrier [13]. These data may be consistent with the clinical benefit observed in our case, which may be explained by a biological activity inside the central nervous system.

\section{Conclusion}

In this case report, we described a rare case of intramedullary spinal cord and leptomeningeal relapse occurring less than 3 years after the initial diagnosis of SCC, which was treated with a combination of systemic cetuximab and intrathecal chemotherapies. The treatment allowed temporary but maintained clinical stabilization with radiological improvement. This case report shows that spinal cord or meningeal metastases must not be ignored if a patient with head and neck SCC develops back pain or neurological disorders. This kind of tumor spread in head and neck cancer is rare and has a poor prognosis. However, the use of epidermal growth factor receptor inhibitors and locally delivered chemotherapy may be effective in such cases.

\section{Disclosure Statement}

The authors declare to have no conflicts of interest regarding the publication of this article.

\section{References}

1 Binder-Foucard F, Belot A, Delafosse P, Woronoff A, Bossard N: Estimation nationale de l'incidence et de la mortalité par cancer en France entre 1980 et 2012. Partie 1 - Tumeurs solides. Institut de veille sanitaire 2013.

2 Redman BG, Tapazoglou E, Al-Sarraf M: Meningeal carcinomatosis in head and neck cancer. Report of six cases and review of the literature. Cancer 1986;58:2656-2661.

-3 Törnwall J, Snäll J, Mesimäki K: A rare case of spinal cord metastases from oral SCC. Br J Oral Maxillofac Surg 2008;46:594-595.

4 Murray JJ, Greco FA, Wolff SN, Hainsworth JD: Neoplastic meningitis. Marked variations of cerebrospinal fluid composition in the absence of extradural block. Am J Med 1983;75:289-294.

5 Sullivan LM, Smee R: Leptomeningeal carcinomatosis from perineural invasion of a lip squamous cell carcinoma. Australas Radiol 2006;50:262-266. 


\section{Case Reports in Oncology}

\begin{tabular}{l|l}
\hline Case Rep Oncol 2014;7:33-38 & \multicolumn{2}{l}{} \\
\hline DOI: $10.1159 / 000358049$ & $\begin{array}{l}\text { C 2014 S. Karger AG, Basel } \\
\text { www.karger.com/cro }\end{array}$ \\
\hline
\end{tabular}

Pougnet et al.: Carcinomatous Myelitis and Meningitis after a Squamous Cell Carcinoma of the Lip

6 Banerjee TK, Gottschalk PG: Unusual manifestations of multiple cranial nerve palsies and mandibular metastasis in a patient with squamous cell carcinoma of the lip. Cancer 1984;53:346-348.

7 Skripuletz T, Pul R, Herrmann J, Bueltmann E, Wurster U, Stangel M, et al: Meningeal carcinomatosis from penile squamous cell carcinoma. J Neurooncol 2010;98:417-419.

-8 Hammerer V, Pauli G, Quoix E: Retrospective study of a series of 26 carcinomatous meningitis secondary to lung cancer (in French). Bull Cancer (Paris) 2005;92:989-994.

-9 Thompson SR, Veness MJ, Morgan GJ, Shannon J, Kench JG: Leptomeningeal carcinomatosis from squamous cell carcinoma of the supraglottic larynx. Australas Radiol 2003;47:325-330.

-10 Lee 0, Cromwell LD, Weider DJ: Carcinomatous meningitis arising from primary nasopharyngeal carcinoma. Am J Otolaryngol 2005;26:193-197.

-11 Emanuel SL, Hughes TV, Adams M, Rugg CA, Fuentes-Pesquera A, Connolly PJ, et al: Cellular and in vivo activity of JNJ-28871063, a nonquinazoline pan-ErbB kinase inhibitor that crosses the blood-brain barrier and displays efficacy against intracranial tumors. Mol Pharmacol 2008;73:338-348.

$\checkmark 12$ Eller JL, Longo SL, Kyle MM, Bassano D, Hicklin DJ, Canute GW: Anti-epidermal growth factor receptor monoclonal antibody cetuximab augments radiation effects in glioblastoma multiforme in vitro and in vivo. Neurosurgery 2005;56:155-162; discussion 162.

-13 Rades D, Nadrowitz R, Buchmann I, Hunold P, Noack F, Schild SE, et al: Radiolabeled cetuximab plus wholebrain irradiation (WBI) for the treatment of brain metastases from non-small cell lung cancer (NSCLC). Strahlenther Onkol Organ Dtsch Röntgengesellschaft Al 2010;186:458-462.

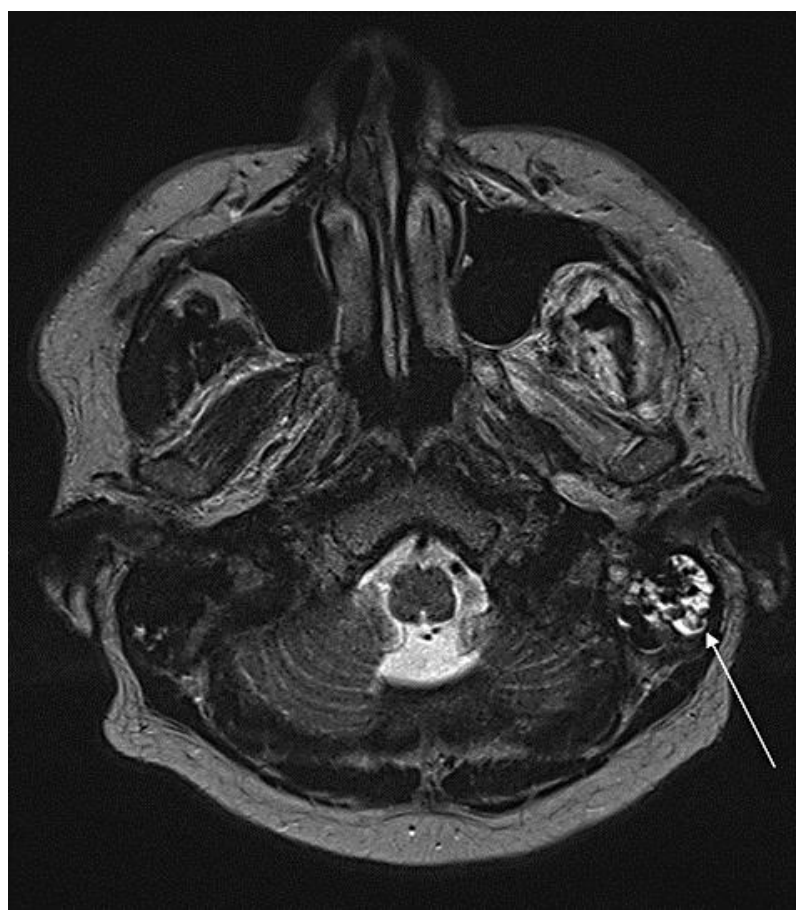

Fig. 1. Facial T2 FLAIR-weighted MRI. Hyperintensity located in the left temporal fossa corresponding to a perineural invasion of the trigeminal nerve (arrow). 


\section{Case Reports in Oncology}

\begin{tabular}{l|l}
\hline Case Rep Oncol 2014;7:33-38 & \multicolumn{2}{l}{$\begin{array}{l}\text { C 2014 S. Karger AG, Basel } \\
\text { www.karger.com/cro }\end{array}$} \\
\hline DOI: 10.1159/000358049 &
\end{tabular}

Pougnet et al.: Carcinomatous Myelitis and Meningitis after a Squamous Cell Carcinoma of the Lip
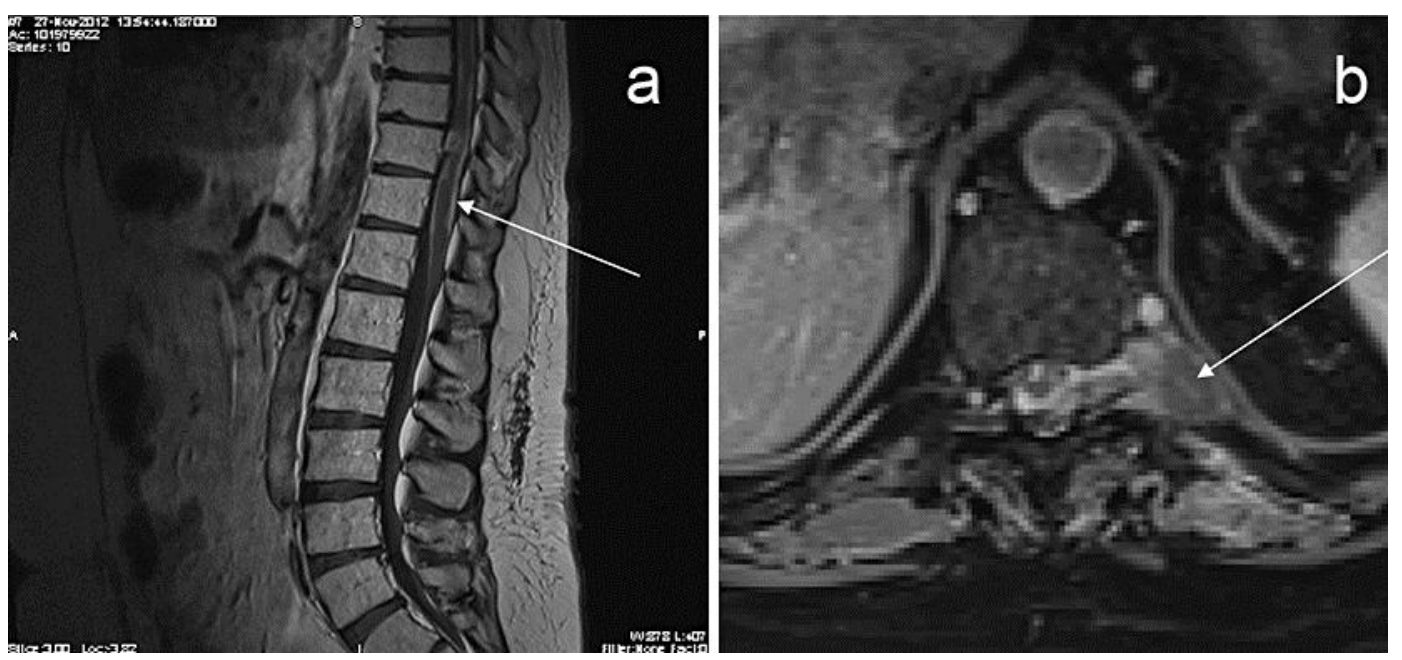

Fig. 2. Spine MRI. a Sagittal plane: diffuse leptomeningeal pathological enhancement after gadolinium injection. b Axial plane: abnormal medullary signal enhancement and left paravertebral invasion (arrow).
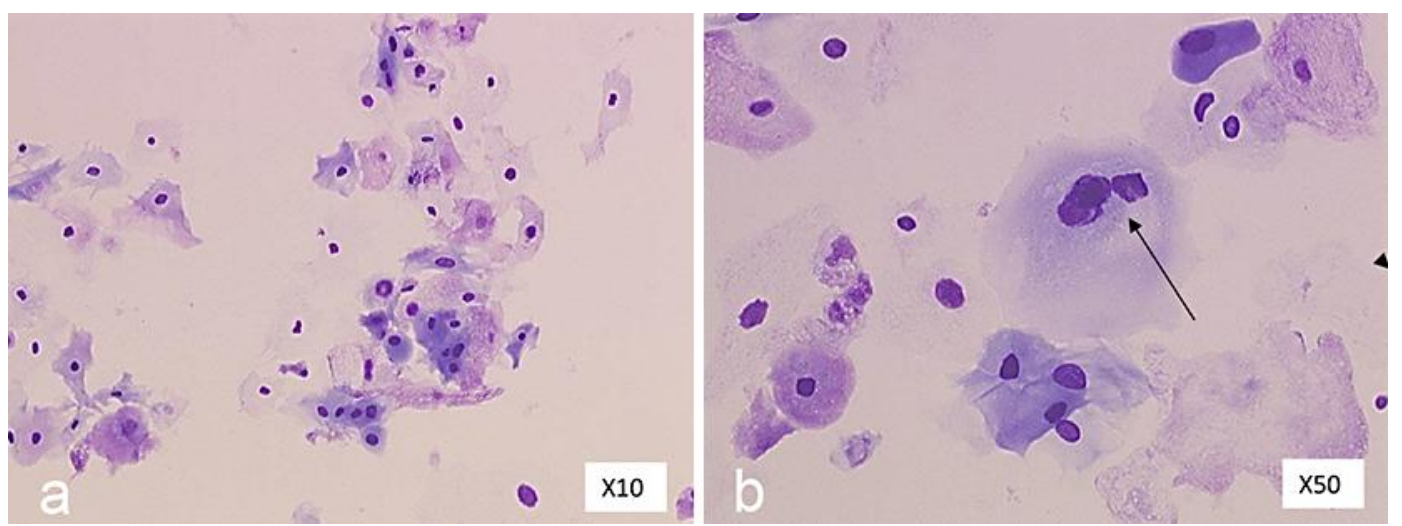

Fig. 3. CSF examination using Cytospin ${ }^{\circledR}$ with May-Grunwald Giemsa staining. a Many well-differentiated squamous cells are observed. b At higher enhancement ( $\times 50)$, few nuclear abnormalities were identified (arrow). 